\title{
Treatment of obese patients with binge eating disorder using topiramate: a review
}

\author{
This article was published in the following Dove Press journal: \\ Neuropsychiatric Disease and Treatment \\ 7 July 2009 \\ Number of times this article has been viewed
}

\author{
Paolo Leombruni \\ Luca Lavagnino \\ Secondo Fassino \\ Department of Neurosciences, \\ Psychiatry Section, University \\ of Torino, Centre for Eating \\ Disorders and Obesity, \\ Torino, Italy
}

Correspondence: Paolo Leombruni Neurosciences Department of Turin University, Psychiatry Section, V Cherasco II, 10126, Turin, Italy Tel +39-0 I I-6634848

Fax+39-0 II-39-0 I I-673473

Email paolo.leombruni@unito.it

\begin{abstract}
Topiramate is an anticonvulsant drug used for the treatment of epilepsy and prophylaxis of migraine. Some authors have proposed its use as a mood stabilizer and have reported its efficacy in reducing impulsiveness and improving mood regulation, possibly via its antagonism to glutamatergic transmission in the lateral hypothalamus, although this indication is still controversial. Weight loss is a side effect consistently reported in the medical literature in patients treated with topiramate. Given its potential role in stabilizing mood and reducing impulse control problems and weight, topiramate has been proposed as a treatment for obese patients with binge eating disorder (BED). The aim of this paper is to review published data on the efficacy and safety of topiramate for the treatment of obese subjects with BED. Although the evidence is preliminary, topiramate appears to be a relatively safe and effective treatment for obese subjects with BED. Limitations of the studies and future directions for research are discussed.
\end{abstract}

Keywords: topiramate, binge eating disorder, obesity

\section{Introduction}

Binge eating disorder (BED) has been the subject of an increasing number of recent studies; nevertheless, it is still awaiting official diagnostic recognition, and it is classified as a syndrome in need of further study in the most recent edition of the Diagnostic and Statistical Manual of Mental Disorders (DSM-IV-TR).

$\mathrm{BED}$ is characterized by recurrent episodes of binge eating (defined as the ingestion of a large quantity of food in a discrete period of time) accompanied by a subjective sense of loss of control over eating. Other criteria are distress about binge episodes; eating more rapidly than normal; eating until uncomfortably full; eating large amounts when not hungry; eating alone because of embarrassment; and feeling disgusted, depressed, or guilty about overeating. In a community survey, $5 \%$ of obese subjects met BED criteria, while $30 \%$ of patients participating in weight loss programs met BED criteria. ${ }^{1}$ It is probably the most frequent eating disorder, ${ }^{2}$ and it is associated with obesity and significant medical and psychiatric comorbidity (especially with regard to anger and personality issues). ${ }^{3-5}$ Some psychotropic medications have also been found to be associated with the induction of binge eating. ${ }^{6}$ These features, together with its chronic nature and its greater prevalence than anorexia and bulimia nervosa, suggest that it should be considered a public health problem of no less importance than the other two more established eating disorders. ${ }^{2}$

Despite a growing recognition of the consequences of BED on public health, substantive evidence on treatment approaches for the disorder is limited. A combination 
of pharmacological and behavioral strategies has been proposed. ${ }^{7}$ Research on the pharmacological treatment of BED has focused mostly on selective serotonin reuptake inhibitors (SSRIs) because BED shares some clinical features with bulimia nervosa. However, a recent meta-analysis ${ }^{8}$ has concluded that currently available data are not sufficient to formally recommend antidepressants as a first-line therapy for BED. The search for novel pharmacological treatments for BED is now focusing on other classes of drugs, particularly sibutramine and topiramate. ${ }^{9}$ Topiramate, which is approved for treatment of epilepsy and prophylaxis of migraine, has been shown consistently to reduce weight in several placebo-controlled obesity trials. ${ }^{10}$ It has also been shown to be effective in treating bulimia nervosa in a randomized controlled trial (RCT). ${ }^{11}$

Current knowledge about the mechanism by which topiramate reduces binge eating is not conclusive. Topiramate might have a specific effect in reducing food intake mediated by its antagonism to glutamatergic transmission in the lateral hypothalamus. ${ }^{12}$ Other possible mechanisms of action reviewed by McElroy and colleagues ${ }^{13}$ include directly inducing weight loss by stimulating energy expenditure, decreasing binge eating by altering the rewarding properties of food, and reducing general impulsivity.

The aim of this paper is to review available evidence on the efficacy/effectiveness and safety of topiramate in adults with BED.

\section{Methods}

We conducted a systematic search using the following computerized literature databases: PubMed, Medline, and PsychInfo. Search terms included "topiramate" and "binge," "binge eating," "obesity," and "binge eating disorder." The search in computerized bibliographic databases was complemented with journal hand-searching.

Given the limited number of studies available, we reviewed RCTs as well as case series and single case reports. The data presented cannot be synthesized with a systematic approach because of the lack of homogeneity among the studies examined; however, the breadth of the selection criteria allows for wide application of these data to different populations of patients with BED.

We restricted our evaluation to studies including adult obese subjects.

\section{Literature review}

The reviewed articles are summarized in Table 1 . We first consider studies involving RCTs and then address those involving open labels. We included both kinds of studies in the belief that the effects of topiramate both under ideal conditions (efficacy studies such as RCTs) and routine clinical situations (effectiveness trials such as those reporting on the effects of topiramate as an add-on to other psychopharmacologic treatments) can contribute to treatment of this disorder.

\section{Randomized controlled trials}

A 14-week, double-blind, flexible dose (25 to $600 \mathrm{mg} /$ day), placebo-controlled trial assessed the efficacy of topiramate in 61 obese [body mass index $(\mathrm{BMI})>30$ ] outpatients with BED (53 women, 8 men). ${ }^{14}$ The study sample consisted of patients aged 18 to 60 years who met DSM-IV-TR criteria for BED and had a score of $>15$ on the Yale-Brown Obsessive Compulsive Scale modified for binge eating (YBOCS-BE). Topiramate was administered at a dose of 100 to $600 \mathrm{mg} /$ day depending on response to treatment. The primary efficacy measure was the number of binge eating episodes during the 7 days before each visit. Secondary measures of efficacy were the number of days in which the patient had at least one binge during the 7 days before each visit, the Clinical and Global Impression improvement (CGI-I) and severity (CGI-S) scales, the YBOCS-BE, and the Hamilton Depression Rating Scale (HDRS or HAM-D). Physical measures included BMI, waist-to-hip ratio, and percent total body fat (measured by bioelectrical impedance). The median dose of topiramate was $212 \mathrm{mg}$ /day (range 50 to 600). Statistical analyses were performed both as intention to treat (with the last observation carried forward) and as completer analysis. Fourteen of 30 patients in the topiramate group (46.7\%) and 12 of 31 patients in the placebo group (38.7\%) did not complete the 14 weeks of treatment. Among the causes for withdrawing from the study were adverse events (topiramate, $\mathrm{n}=6$, placebo, $\mathrm{n}=3$; mainly headache, paresthesia, amenorrhea, leg cramps, and sedation), lack of efficacy (topiramate, $n=1$, placebo, $n=2$ ), nonadherence to the study protocol (topiramate, $n=6$; placebo, $n=7$ ), and exacerbation of a preexisting medical condition (topiramate, $\mathrm{n}=1$ ). Patients receiving topiramate achieved greater reductions in binge frequency, which was the primary outcome measure, than those in the placebo group ( $94 \%$ vs $46 \%$, respectively, $P<0.002)$, as well as reductions in binge day frequency (93\% vs $46 \%, P<0.02)$, CGI score $\left(\chi^{2}=6.31, P=0.01\right)$, weight loss (5.9 kg vs $1.2 \mathrm{~kg})$, and reduction of body fat (reduction in percent body fat: $\chi^{2}=7.87, P=0.006$; reduction in total body fat: $\left.\chi^{2}=10.68, P=0.001\right)$. Limitations included the short study period and the high percentage of 


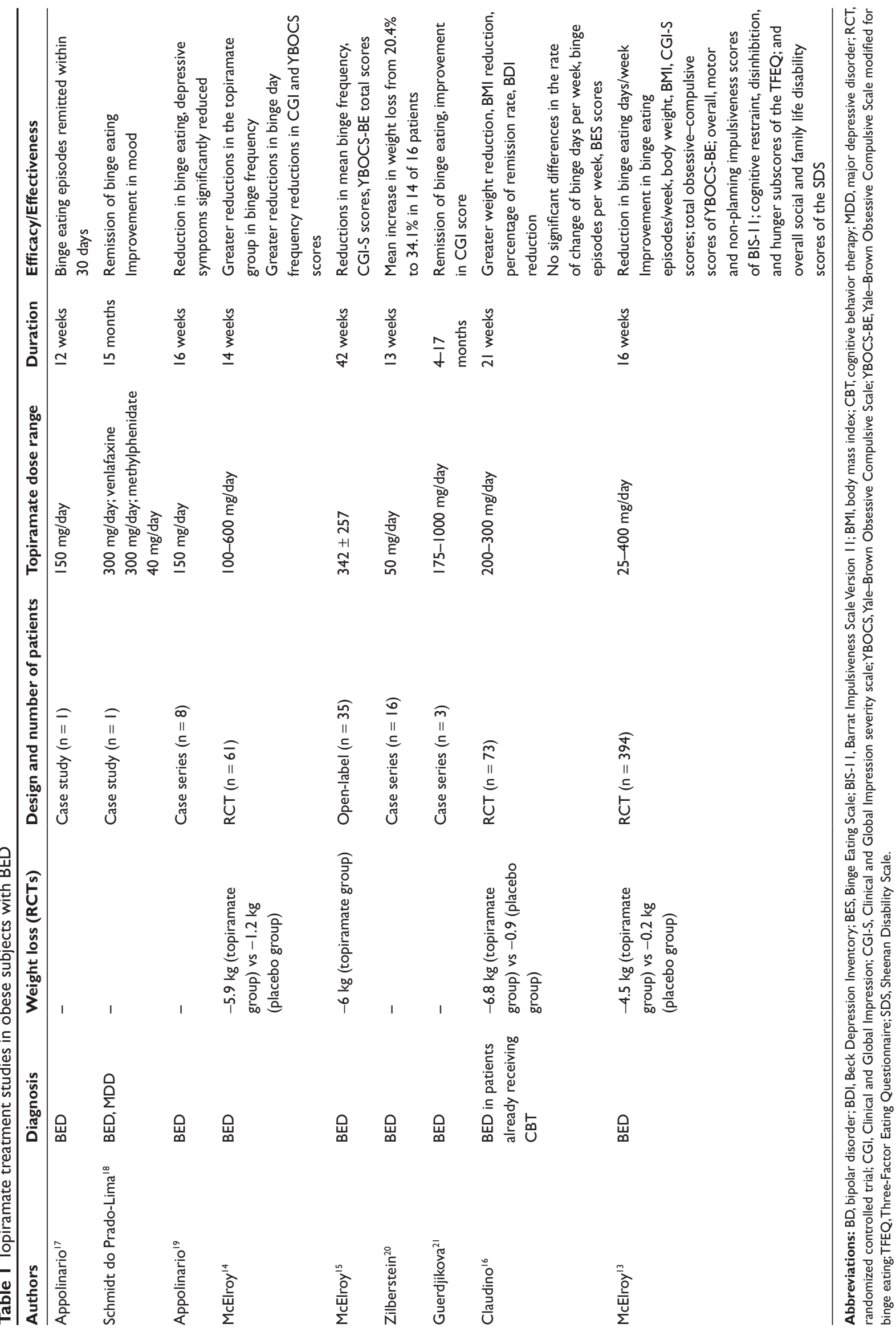


dropouts among the patients. The authors concluded that topiramate was effective in reducing binge eating behavior and weight.

The authors of the previous study performed a 42-week, open-label extension to assess the long-term efficacy, tolerability, and safety of topiramate in BED associated with obesity. ${ }^{15}$ This study will be discussed in this section because it represents the continuation of the previous study. Thirty-five patients completed the controlled study (topiramate, $n=15$; placebo, $\mathrm{n}=16) ; 31$ of these were interested in pursuing open-label treatment with topiramate and were enrolled in the extension study. The primary endpoint was change from baseline to the final visit in weekly binge frequency using the last observation carried forward. Some patients received double-blind topiramate from the beginning of the controlled study; the outcome for this group was measured in terms of changes in binge frequency since the beginning of the study. Baseline binge frequency was defined as the frequency of bingeing at the beginning of treatment with topiramate for patients who received placebos in the double-blind period of the study. Topiramate was titrated from an initial dose of $25 \mathrm{mg} /$ day to a maximum dose of $600 \mathrm{mg} /$ day, as needed, to achieve a reduction in binge frequency and as tolerated. Outcome measures considered were the same as for the double-blind trial. Efficacy analyses were performed on an intent-to-treat basis for all patients, with at least one efficacy evaluation after double-blind or open-label topiramate treatment. Forty-four patients received at least one dose of topiramate during the double-blind $(\mathrm{n}=13)$ or open-label extension period $(n=31)$. One patient never returned for an evaluation after taking topiramate; thus 43 patients were included in the final analysis. The median duration of therapy among these patients was 23.5 weeks. Thirty-five patients discontinued topiramate during the controlled study $(n=14)$ or the extension trial $(n=21)$. The mean dose of topiramate administered was $342 \pm 257 \mathrm{mg} /$ day. Ten patients completed a total of 56 weeks of treatment. The intent-to-treat analysis revealed that patients treated with topiramate during either the controlled or extension studies achieved significant reductions in mean binge frequency $(\mathrm{n}=43,-3.2, P<0.001)$, which were even more evident in patients who completed treatment $(\mathrm{n}=10,-5.0, P=0.002)$. Analysis of secondary outcomes showed significant reductions from baseline CGI-S scores $(P<0.001)$, YBOCS-BE total scores $(P<0.001)$, body weight $(-6.0 \mathrm{~kg}, P<0.001)$, and BMI $\left(-2.1 \mathrm{~kg} / \mathrm{m}^{2}\right.$, $P<0.001)$. The mean Hamilton Rating Scale for Depression (HAM-D or HDRS) scores were not significantly reduced from baseline to the final visit. Fourteen of 44 patients discontinued topiramate because of adverse events. The most frequently reported adverse events were paresthesia (75\% of patients), dry mouth (46\%), headache (36\%), taste disturbance (34\%), cognitive problems (32\%), dizziness (30\%), somnolence (25\%), fatigue (25\%), dyspepsia $(25 \%)$, diarrhea (21\%), confusion (18\%), nausea (18\%), and nervousness $(16 \%)$. Patients who discontinued topiramate because of adverse effects were not taking a higher dose than those who discontinued topiramate for other reasons $(225 \pm 179$ vs $326 \pm 282 \mathrm{mg} /$ day $)$. The severity of these adverse events was considered mild to moderate. The authors concluded that subjects with BED continued to experience a reduction in binge eating and had further weight loss with topiramate treatment during the open-label extension of the study. Limitations regarding the design of this study are the lack of a control group and randomization, and the fact that it was open-label; other limitations include the small sample size and the high attrition rate.

McElroy and colleagues ${ }^{13}$ investigated the safety and efficacy of topiramate in a 16-week, multicenter, randomized placebo-controlled trial. Patients were men and women between 18 and 65 years of age with a diagnosis of BED, having at least 3 binge days per week and a BMI of 30 to $50 \mathrm{~kg} / \mathrm{m}^{2}$. Patients with a history of bipolar disorder, psychotic disorder, substance abuse, current clinically significant depression, and those with a BMI $>50 \mathrm{~kg} / \mathrm{m}^{2}$ were excluded to reduce the rate of discontinuation attributable to decreased impulse control and poor health. Patients were randomized to receive topiramate (195 patients) or placebo (199 patients). The primary outcome measure was the number of binge eating days per week. Secondary outcome measures included binge eating episodes per week, body weight, BMI, CGI score, YBOCS-BE score, Barrat Impulsiveness Scale Version 11 (BIS-11), Three-Factor Eating Questionnaire (TFEQ), Hamilton Anxiety Scale (HAM-A), and Sheenan Disability Scale (SDS). The median dose of topiramate was $300 \mathrm{mg}$ (range 25 to $400 \mathrm{mg} /$ day). Patients treated with topiramate achieved a significantly greater rate of reduction in binge eating days per week than those receiving placebo (from 4.6 to 0.9 vs from 4.6 to $2.2 ; \chi^{2}=51, P<0.001$ ). Topiramate was also associated with a significantly greater improvement than placebo for binge eating episodes per week; body weight; BMI; CGI-S scores; total obsessivecompulsive scores of the YBOCS-BE scale; overall motor and non-planning impulsiveness scores of the BIS-11 scale; cognitive restraint, disinhibition, and hunger subscores of the TFEQ; and overall social and family life, and disability scores of the SDS. On the other hand, between-group differences 
were not found in the rate of change in the Montgomery Asberg Depression Rating Scale (MADRS), HAM-A, SDS school/work disability, or BIS-11 attentional impulsiveness scores. Fifty-eight percent of patients treated with topiramate achieved remission, whereas only $29 \%$ of patients treated with placebo remitted $(P<0.001)$. Adverse events reported by more than $10 \%$ in the topiramate group were paresthesia, upper respiratory tract infection, taste disturbance, difficulty with concentration/attention, and difficulty with memory not otherwise specified. Most adverse events were mild to moderate in severity; the most common adverse events causing topiramate discontinuation were difficulty with memory not otherwise specified and depression. Twenty-nine $(15 \%)$ discontinuations in the topiramate group were attributable to adverse events. Limitations of this study include the use of self-report measures, short study duration, risk that the blinding procedure might have been spoiled by the higher prevalence of adverse events in topiramate-treated patients compared to the placebo group, and exclusion of patients with Axis I psychiatric disorders, which may make it impossible to generalize the results of the study to patients with significant psychiatric comorbidity. The authors concluded that topiramate was efficacious and well tolerated in the treatment of obese patients with BED.

Claudino and colleagues ${ }^{16}$ evaluated the efficacy of adjunctive treatment with topiramate compared to that with placebo in reducing weight gain and binge eating in obese patients receiving cognitive behavior therapy (CBT). This study consisted of a 21-week double-blind, randomized, and placebo-controlled trial. The sample consisted of 73 adults aged 18 to 60 years ( 70 women, 3 men) with a BMI $\geq 30 \mathrm{~kg} / \mathrm{m}^{2}$ who met DSM-IV-TR criteria for BED and scored 17 or more on the Binge Eating Scale (BES). Patients were randomly assigned to receive topiramate or placebo with doses titrated to $200 \mathrm{mg} /$ day. Doses were increased to a maximum of $300 \mathrm{mg} /$ day in patients who failed to achieve a reduction in baseline weight of at least $5 \%$ or a reduction in the number of days with binge episodes of at least $50 \%$. Dose reductions were allowed for patients who did not tolerate their current doses. The primary outcome measure was weight change; secondary efficacy measures were BMI, binge frequency, BES scores, depressive symptoms (Beck Depression Inventory [BDI] scores), and treatment tolerance. At the end of the study period, the mean weight change was $-6.8 \mathrm{~kg}$ in the topiramate group vs $-0.9 \mathrm{~kg}$ in the placebo group. Patients randomized to topiramate showed a significantly greater rate of weight reduction (topiramate $\times$ time interaction) than patients assigned to the placebo group $\left(\chi^{2}=16.1, P<0.001\right)$ and a final BMI of $35 \pm 3.5 \mathrm{~kg} / \mathrm{m}^{2}$, whereas those taking the placebo demonstrated a final BMI of $36.7 \pm 4.7 \mathrm{~kg} / \mathrm{m}^{2}$. The percent weight loss was also greater in topiramate-treated patients than in those receiving the placebo $(6.8 \pm 5.2 \%$ vs $4.1 \pm 4.3 \%, P=0.04)$. At the end of the trial, a greater percentage of patients receiving topiramate attained a weight loss of $>10 \%$ compared to those receiving placebo $(33.3 \%$ vs $\left.11.5 \% ; \chi^{2}=3.71, P=0.05\right)$. No significant differences were found between treatment groups with regard to the rate of change in the number of binge days per week, the number of binge episodes per week, or BES scores. The rate of remission for binge eating in the intent-to-treat group was significantly higher among patients taking topiramate (83.8\%) than among those taking placebo (61.1\%). BDI scores were significantly reduced in both treatment groups but these changes did not reach statistical significance $(P=0.20)$. Seventeen patients $(23.3 \%$ of the sample) dropped out of treatment ( 7 in the topiramate group, 10 in the placebo group). The most frequent cause for withdrawing from treatment was nonadherence to the study protocol. Only one patient in the topiramate group withdrew because of adverse events, which consisted of paresthesia and confusion, probably due to the patient taking the wrong dose of medication (the exact dose was not reported in the article). The adverse events most commonly reported by patients receiving topiramate were paresthesia (48.6\%), taste disturbance $(24.3 \%)$, dysuria $(13.5 \%)$, and leg pain $(10,8 \%)$. The authors highlight the efficacy of the combination of topiramate and CBT in producing weight reduction and significant remission in bingeing in obese patients with BED.

\section{Open-label studies}

Appolinario and colleagues ${ }^{17}$ described the effects of topiramate on a woman diagnosed with BED in the absence of neuropsychiatric comorbidity. This woman had reported engaging in eight binge episodes each week and had previously been treated with amphetamine-like drugs, fluoxetine, and sibutramine without benefit. The initial topiramate dose was $25 \mathrm{mg}$ bid for 2 weeks, followed by an increase of $25 \mathrm{mg}$ every week until she reached the target dose of $75 \mathrm{mg}$ bid, which was maintained for 4 months. After the first 2 weeks of treatment, weekly binge eating decreased from 8 to 1 , and the episodes completely remitted within 30 days of the onset of treatment. Her respective weight and BMI, which were $109 \mathrm{~kg}$ and $42.5 \mathrm{~kg} / \mathrm{m}^{2}$ before starting treatment, decreased to $98.6 \mathrm{~kg}$ and $38.5 \mathrm{~kg} / \mathrm{m}^{2}$ after 4 months of treatment. The only reported side effect was somnolence at the beginning of treatment. The authors did not use rating scales or standardized measures. 
Schmidt do Prado-Lima and Bacaltchuck ${ }^{18}$ described the topiramate treatment of a patient with refractory unipolar depression and comorbid BED. Topiramate was added to a drug regimen consisting of methylphenidate $(20 \mathrm{mg} /$ day $)$ and venlafaxine $(150 \mathrm{mg} /$ day $)$, and was titrated upward until a dose of $300 \mathrm{mg} /$ day was reached. The patient experienced a remission in binge eating and a significant improvement in mood. Because this study used a single case characterized by comorbidity and polypharmacy, the results are difficult to generalize.

Appolinario and colleagues ${ }^{19}$ assessed the efficacy and tolerability of topiramate in a group of obese binge eaters with no neuropsychiatric comorbidity in a 16-week, open-label study. The study sample included eight female outpatients with a BMI $>30$, a diagnosis of BED according to DSM-IV criteria, and at least moderately severe binge eating behavior, defined as a BES score of $>17$. The dose of topiramate was gradually increased in weekly increments of $25 \mathrm{mg}$ from $25 \mathrm{mg}$ twice daily during the first 2 weeks to the target dosage of $75 \mathrm{mg}$ twice daily at 2 months. Outcome measures included the number of days per week containing binge eating episodes (DBEs), BES scores, scores on the BDI, body weight, and adverse events. In reporting outcomes, an intent-to-treat analysis was employed. Of the eight patients enrolled in the study, 6 had attended all scheduled visits, 4 patients displayed a total remission of binge eating episodes, and 2 demonstrated a marked reduction in binge frequency. Two patients discontinued the trial, 1 because of diurnal somnolence at visit two and 1 due to lack of efficacy at week 6 . Reported side effects were transient and included paresthesia (the most common, experienced by 4 patients), fatigue, somnolence, psychomotor slowing, impaired concentration, and nausea. All patients who completed the trial showed a reduction in binge eating (DBEs were reduced from $4.3 \pm 1.7$ to $1.1 \pm 2.4, P=0.03$; BES scores decreased from $31.8 \pm 7.5$ to $15.3 \pm 9.2, P=0.04)$; a statistically significant weight loss was observed (mean $4.1 \mathrm{~kg}, P=0.04$ ) and depressive symptoms decreased significantly (mean BDI scores declined from $25.3 \pm 7.5$ to $15.8 \pm 5.7, P=0.02$ ). The authors concluded that topiramate was effective in reducing eating psychopathology. Limitations of this study are its openlabel design, small sample size, and the impossibility of distinguishing between response to treatment and placebo effects, which is known to be quite high in BED.

Zilberstein and colleagues ${ }^{20}$ reported on the use of topiramate for BED in 16 patients who stopped losing weight after adjustable gastric banding. Topiramate was given in doses varying from 12.5 to $50 \mathrm{mg}$ /day. The authors reported a mean increase in weight loss from $20.4 \%$ to $34.1 \%$ in 14 patients after 90 days, without the need for band readjustment. Two patients were reported to have intolerance to topiramate; no further details were provided about the kinds of symptoms experienced.

Other anecdotal observations on the use of topiramate in three patients who underwent bariatric surgery were reported by Guerdjikova and colleagues. ${ }^{21}$ These patients gained weight and showed recurrent binge eating after initially successful bariatric surgery. The dose of topiramate prescribed ranged from 175 to $1000 \mathrm{mg}$ /day. They reported remission of binge eating and displayed significant weight loss $(31.7 \mathrm{~kg}, 14.5 \mathrm{~kg}$, and $2 \mathrm{~kg}$ in 17,9 , and 4 months, respectively).

\section{Conclusions and future directions}

The existing literature appears to suffer from some limitations. The first concern that emerges from analyzing studies on the use of topiramate for BED is the long-term effects of this treatment. Obesity is known to be a chronic health problem, and long-term relapses after initial weight loss are known to be frequent; nevertheless, only one of the controlled studies reviewed ${ }^{15}$ involved long-term follow-up (42 weeks). This suggests caution in interpreting data, as the effects of long-term treatment with topiramate have not been extensively investigated.

A second limitation of the studies reviewed is the fairly high dropout rate of up to $30 \%$. Adverse events have been discussed as a cause of patient dropout, but other causes (eg, protocol nonadherence) have not been discussed extensively. The acceptability of drug treatment by BED patients probably depends on many factors, among which the expectations of patients and motivation to change are likely to play a major role. It is noteworthy that completion rates were higher in the study of Claudino and colleagues ${ }^{16}$ in which CBT was part of the study design. Appropriate strategies (counseling and psychotherapy) should be tested for their effectiveness in leading patients to more realistic weight loss goals and in sustaining motivation for drug adherence and lifestyle change.

A third issue is in regard to the wide exclusion criteria used by most RCTs. Frequent comorbidity (such as severe depression, bipolar disorder, or personality disorders) may lower the likelihood of remission of binge eating in the broad population of binge eaters compared to that of the patients considered in the trials described here. On the other hand, comorbidity can also provide indications that 
are valuable for targeting the pharmacological treatment to the needs of the individual patient (eg, treating a patient having bipolar symptoms with a mood stabilizer instead of an antidepressant) and should therefore be addressed in clinical research.

Another element that creates some difficulty in the interpretation of available data is the lack of homogeneity in psychological testing for the assessment of binge eating. Scales used in the studies reviewed include the Eating Disorder Inventory, second edition (EDI-2), BES, and YBOCS-BE. A consensus on which assessment tool should be used would add to the comparability of the published studies.

Note that two of the three RCTs were sponsored by the manufacturer of topiramate; these two studies reported better results for topiramate than placebo in reducing binge eating, whereas the third RCT did not find a significant effect for this drug. These results should be replicated by other independent studies to provide further evidence for the use of topiramate in BED.

A final limitation is in regard to subthreshold binge eaters. Some authors have contended that patients who feel a subjective sense of loss of control over eating might present with a level of distress and functional impairment that is comparable to that characteristic of eating disorders, even if they do not eat large amounts of food in a discrete period of time, as required by the DSM-IV-TR definition of a binge episode. ${ }^{22}$ This category of patients is probably subject to the same hazards to health and well-being as those who satisfy all the criteria for BED. Although these patients constitute a large proportion of overweight patients who fail several diet regimens and attend visits with nutritionists and eating disorders centers, indications about the role of psychopharmacological treatment in this population are just beginning to emerge. ${ }^{23}$

In conclusion, topiramate appears to be a relatively safe and effective treatment for obese subjects with BED. The drug should probably be started at $25 \mathrm{mg}$ /day and increased by $25 \mathrm{mg}$ /day every 1 to 2 weeks, until reaching a dose of 150 to $200 \mathrm{mg} /$ day. It could then be further increased up to a dose of $400 \mathrm{mg} /$ day or more for selected patients in the absence of clinical response.

Graphic representations of responses shown in the most comprehensive studies revealed that clinically significant changes in binge eating days per week and binge eating episodes per week should be expected after about 4 weeks of treatment, whereas effects on weight might be expected after approximately 6 to 8 weeks.
Topiramate appears to have a weight loss effect greater than those of other effective and well studied treatments for this disorder such as CBT, interpersonal therapy, and SSRIs, and one comparable to that of sibutramine. ${ }^{13}$ As noted above, depressive symptoms are frequently associated with BED, but as yet no compelling evidence exists of the efficacy of topiramate on this symptom dimension. This is partly a consequence of excluding patients with severe comorbid psychopathology from BED trials. The presence of moderate to severe depressive symptoms might be an indication for antidepressant treatment for binge eating patients.

Some authors have raised doubts about the tolerability of topiramate, drawing on data from the epilepsy literature; however, the dosages used in BED patients seem to lead to a somewhat milder adverse effect profile. The risk of kidney stones has been associated with this drug, but no cases of this adverse effect have been reported in obese patients with BED. Topiramate has been included in risk category 3 regarding its use in pregnancy. This means that some animal studies have shown adverse effects; no controlled studies in humans have been conducted, although some case reports of hypospadia in male infants whose mothers took topiramate during pregnancy have been reported.

In this context, the use of topiramate in women with childbearing potential should be decided after weighing the potential benefits to the mother against the potential risks to the fetus. Further research is required regarding the long-term effects and the optimal dosing in BED patients. It will also be relevant to perform direct comparisons of the benefits and adverse effects of topiramate with those of other drugs that have been shown to be effective in reducing both binge eating and body weight in controlled trials, such as sibutramine, zonisamide, and atomoxetine. Future studies should focus on the indications for and effects of pharmacotherapy and evidence-based psychological treatments (such as CBT) for eating disorders as both separate and as combined interventions because the optimal management of BED can be achieved only with a multidisciplinary and multimodal treatment approach. ${ }^{24}$ One of the studies reviewed provides a model for this approach. ${ }^{16}$

\section{Disclosures}

The authors disclose no conflicts of interest.

\section{References}

1. De Zwaan M. Binge eating disorder and obesity. Int J Obes Relat Metab Disord. 2001;25 Suppl 1:S51-S55.

2. Hudson JI, Hiripi E, Pope HG Jr, Kessler RC. The prevalence and correlates of eating disorders in the National Comorbidity Survey Replication. Biol Psychiatry. 2007;61(3):348-358. 
3. Javaras KN, Pope HG, Lalonde JK, et al. Co-occurrence of binge eating disorder with psychiatric and medical disorders. J Clin Psychiatry. 2008;69(2):266-273.

4. Grucza RA, Przybeck TR, Cloninger CR. Prevalence and correlates of binge eating disorder in a community sample. Compr Psychiatry. 2007;48(2):124-131.

5. Fassino S, Leombruni P, Pierò A, Abbate-Daga G, Giacomo Rovera G. Mood, eating attitudes, and anger in obese women with and without Binge Eating Disorder. J Psychosom Res. 2003;54(6):559-566.

6. Kluge M, Schuld A, Himmerich H, et al. Clozapine and olanzapine are associated with food craving and binge eating: results from a randomized double-blind study. J Clin Psychopharmacol. 2007;27(6): 662-666.

7. National Institute for Clinical Excellence. Eating disorders - core interventions in the treatment and management of anorexia nervosa, bulimia nervosa, and related eating disorders. NICE Clinical Guideline No 9, NICE, London; 2004. Available at: http://www.nice.org.uk.

8. Stefano SC, Bacaltchuk J, Blay SL, Appolinario JC. Antidepressants in short-term treatment of binge eating disorder: systematic review and meta-analysis. Eat Behav. 2008;9:129-136.

9. Yager J. Binge eating disorder: the search for better treatments. Am J Psychiatry. 2008;165(1):4-6.

10. Li Z, Maglione M, Tu W, et al. Meta-analysis: pharmacologic treatment of obesity. Ann Intern Med. 2005;142(7):532-546.

11. Nickel C, Tritt K, Muehlbacher M, et al.Topiramate treatment in bulimia nervosa patients: a randomized, double-blind, placebo-controlled trial. Int J Eat Disord. 2005;38(4):295-300.

12. Tata AL, Kockler DR. Topiramate for binge-eating disorder associated with obesity. Ann Pharmacother. 2006;40(11):1993-1997.

13. McElroy SL, Hudson JI, Capece JA, et al; Topiramate Binge Eating Disorder Research Group. Topiramate for the treatment of binge eating disorder associated with obesity: a placebo-controlled study. Biol Psychiatry. 2007;61:1039-1048.
14. McElroy SL, Arnold LM, Shapira NA, et al. Topiramate in the treatment of binge eating disorder associated with obesity: a randomized, placebocontrolled trial. Am J Psychiatry. 2003;160:255-261.

15. McElroy SL, Shapira NA, Arnold LM, et al. Topiramate in the long-term treatment of binge-eating disorder associated with obesity. J Clin Psychiatry. 2004;65:1463-1469.

16. Claudino AM, de Oliveira IR, Appolinario JC, et al. Double-blind, randomized, placebo-controlled trial of topiramate plus cognitive-behavior therapy in binge-eating disorder. J Clin Psychiatry. 2007;68(9): 1324-1332.

17. Appolinario JC, Coutinh W, Fontenelle L. Topiramate for binge-eating disorder. Am J Psychiatry. 2001;158 (6):967-968.

18. Schmidt do Prado-Lima PA, Bacaltchuck J. Topiramate in treatmentresistant depression and binge-eating disorder. Bipolar Disord. 2002;4(4):271-273.

19. Appolinario JC, Fontenelle LF, Papelbaum M, Bueno JR, Coutinho W. Topiramate use in obese patients with binge eating disorder: an open study. Can J Psychiatry. 2002;47(3):271-273.

20. Zilberstein B, Pajecki D, Garcia de Brito AC, Gallafrio ST, Eshkenazy R, Andrade CG. Topiramate after adjustable gastric banding in patients with binge eating and difficulty losing weight. Obes Surg. 2004;14(6):802-805

21. Guerdjikova AI, Kotwal R, McElroy SL. Response of recurrent binge eating and weight gain to topiramate in patients with binge eating disorder after bariatric surgery. Obes Surg. 2005;15(2):273-277.

22. Latner JD, Clyne $C$. The diagnostic validity of the criteria for binge eating disorder.Int J Eat Disord. 2008;41(1):1-14.

23. Leombruni P, Pierò A, Brustolin A, et al. A 12 to 24 weeks pilot study of sertraline treatment in obese women binge eaters. Hum Psychopharmacol. 2006;21(3):181-188.

24. Halmi KA. The multimodal treatment of eating disorders. World Psychiatry. 2005;4(2):69-73.
Neuropsychiatric Disease and Treatment

\section{Publish your work in this journal}

Neuropsychiatric Disease and Treatment is an international, peerreviewed journal of clinical therapeutics and pharmacology focusing on concise rapid reporting of clinical or pre-clinical studies on a range of neuropsychiatric and neurological disorders. This journal is indexed on PubMed Central, the 'PsycINFO' database and CAS, and is the official

\section{Dovepress}

journal of The International Neuropsychiatric Association (INA). The manuscript management system is completely online and includes a very quick and fair peer-review system, which is all easy to use. Visit http://www.dovepress.com/testimonials.php to read real quotes from published authors. 